\title{
Gaseous nitric oxide for the local treatment of bacterial keratitis in mice
}

\author{
MONA DEICHELBOHRER ${ }^{1}$, MING-FENG WU ${ }^{2}$, BERTHOLD SEITZ ${ }^{2}$, STEFAN WAGENPFEIL ${ }^{3}$, \\ CAROLA MEIER $^{1}$, MARKUS BISCHOFF ${ }^{4}$ and THOMAS TSCHERNIG ${ }^{1}$ \\ ${ }^{1}$ Institute of Anatomy and Cell Biology, Saarland University Faculty of Medicine; ${ }^{2}$ Department of Ophthalmology, \\ Saarland University Medical Center; ${ }^{3}$ Institute for Medical Biometry, Epidemiology and Medical \\ Informatics, Saarland University; ${ }^{4}$ Institute of Medical Microbiology and Hygiene, \\ Saarland University Faculty of Medicine, Homburg, D-66424 Saar, Germany
}

Received October 10, 2016; Accepted November 11, 2016

DOI: $10.3892 /$ br.2016.821

\begin{abstract}
The successful treatment of severe bacterial keratitis continues to be a challenge in animals and humans. In the present study the aim was to assess gaseous therapy using gaseous nitric oxide ( $\mathrm{gNO}$ ) in a murine model of Pseudomonas aeruginosa keratitis. The cornea of anesthetized mice was mechanically scratched and covered with a bacterial suspension of $P$. aeruginosa. One day later, the infected eyes were exposed to $200 \mathrm{ppm}$ NO for $30 \mathrm{~min}$. Three to seven days later the mice were sacrificed and the bulbi were obtained and processed for light microscopy. The read out parameter was the maximal corneal thickness and the severity of the hypopyon. The therapy with NO did not result in either a reduction of inflammation concerning the maximal corneal thickness or the severity of the hypopyon. The bacterial load was not investigated due to technical limitations. Thus, exposure to gNO did not reduce the local inflammation in $P$. aeruginosa induced murine keratitis at the investigated time-points. This does not exclude effects of NO on the bacterial load, and in experimental and human keratitis.
\end{abstract}

\section{Introduction}

Bacterial keratitis is a major cause of blindness worldwide with a high incidence (1). This is due to low hygiene standards and water quality with a greater risk of infection in poor countries. In addition, the widespread use of contact lenses and associated complications in wealthier countries contributes to severe keratitis (2,3). Staphylococcus aureus and Pseudomonas aeruginosa are two predominant gram-positive and gram-negative bacterial strains responsible for causing this disease $(1,4,5)$. The

Correspondence to: Professor Thomas Tschernig, Institute of Anatomy and Cell Biology, Saarland University Faculty of Medicine, 61 Kirrberger Strasse, Homburg, D-66424 Saar, Germany

E-mail: thomas.tschernig@uks.eu

Key words: keratitis, gaseous nitric oxide, treatment resistance of bacteria to antibiotics is reducing the efficacy of bacterial keratitis treatment. Therefore, research into the development of novel therapeutic approaches is essential. In this regard photodynamic inactivation has been in experimental and clinical use throughout the last decade (6-8). Research on cystic fibrosis established an antimicrobial effect of gaseous nitric oxide (gNO) in infections with $P$. aeruginosa using a rat model (9). Furthermore, a study in white rabbits demonstrated that a treatment using 200 ppm gNO significantly reduced the bacterial load in wounds of the dorsal skin surface (10). Thus, in view of the possibility of a surface treatment for keratitis, this therapeutic strategy demonstrated a feasible approach.

The aim of the current study was to evaluate gNO application as a potential treatment of experimental keratitis in vivo for the first time. However, this series of experiments did not serve as proof of the anti-inflammatory effect of $\mathrm{gNO}$.

\section{Materials and methods}

Animals and anaesthesia. Young (age, 9-10.5 weeks) female C57BL/6 mice $[\mathrm{n}=19$; standard housing conditions (3-5 animals per cage) with 12-h light/dark cycle, minimum $70 \%$ humidity and chow/water were provided ad libitum; Janvier Labs, Saint-Berthevin Cedex, France] were used in the present study. All animal care and experimental procedures were approved by the local governmental animal care committee (permit no. 58/2013) and were conducted in accordance with European legislation on the protection of animals (Directive 2010/63/EU) and National Institutes of Health (NIH) guidelines on the care and use of laboratory animals (NIH publication no. 85-23 Rev. 1985). Animals were anesthetized via i.p. injection of ketamine (75 mg kg-1 body weight; Pharmacia GmbH, Erlangen, Germany) and xylazine (15 mg kg-1 body weight; Rompun, Bayer, Leverkusen, Germany).

Infection and treatment. Three scratches (length, $2 \mathrm{~mm}$ ) were made in the centre of the cornea using a 27-gauge syringe, subsequently $5 \mu \mathrm{l}$ of the inoculum was pipetted onto the cornea [according to (4)]. Carprofen (Rimadyl ${ }^{\circledR}$; Zoetis Schweiz $\mathrm{GmbH}$, Zürich, Switzerland) was injected during anaesthesia. For the inoculum, the multi drug resistant $P$. aeruginosa 


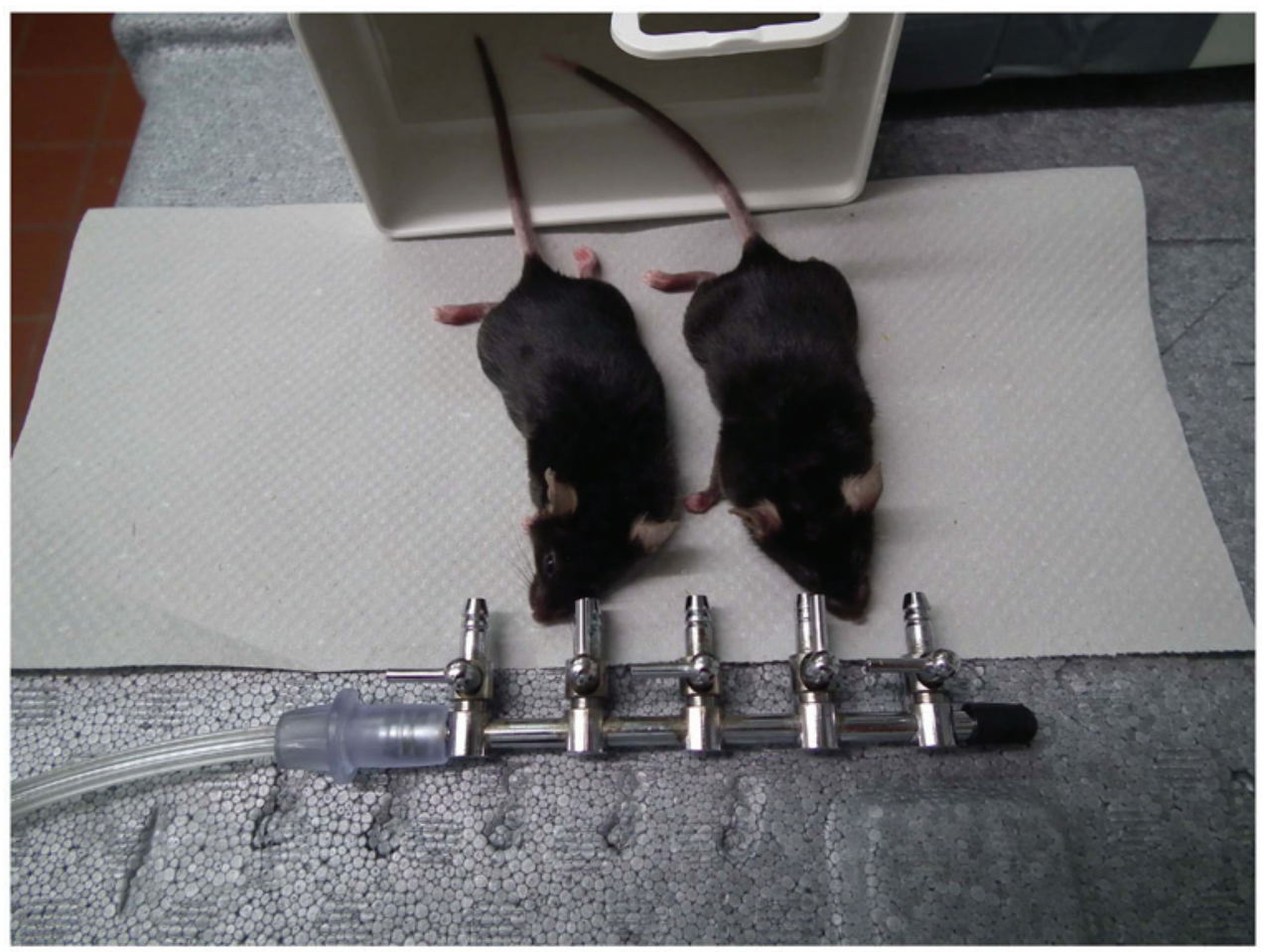

Figure 1. Application of gaseous nitric oxide was performed as a gas flow directed at the infected eye of the mice.

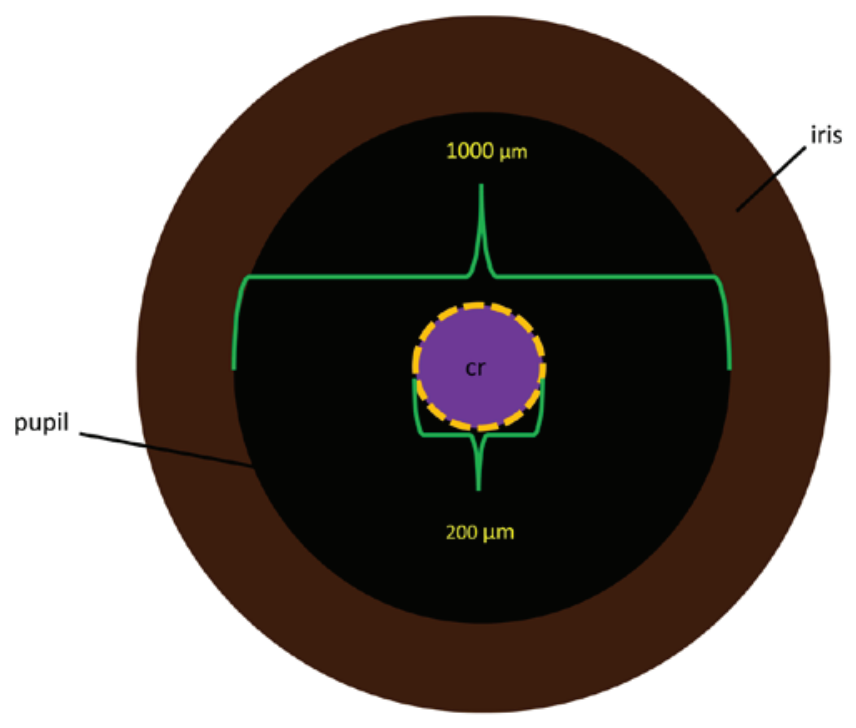

Figure 2. Schematic for obtaining the histological sections. The diameter of the pupil was $\sim 1,000 \mu \mathrm{m}$. The sections were obtained from the $\mathrm{CR}$ of the cornea, which had a diameter of $\sim 200 \mu \mathrm{m}$. CR, centre region.

strain 54 (clinical isolate, collected in 2009 at the Institute of Medical Microbiology and Hygiene, Saarland University Medical Center, Homburg, Germany. The resistance profile of PA54 was determined using the automated VITEK 2 system (BioMérieux GmbH, Nürtingen, Germany) was cultured on blood agar plates (Trypticase Soy Agar II, 5\% Sheep Blood; $\mathrm{BD} \mathrm{GmbH}$, Heidelberg, Germany) for $24 \mathrm{~h}$, and suspended in $10 \mathrm{ml}$ 2\% Luria Bertani (LB) broth (LB Broth; Difco, Saint Egrève, France) for an overnight culture $\left(37^{\circ} \mathrm{C}\right)$. The overnight culture $(100 \mu \mathrm{l})$ was diluted in $10 \mathrm{ml} 2 \% \mathrm{LB}$ for another $3 \mathrm{~h}$ of culturing $\left(37^{\circ} \mathrm{C}\right)$ and finally the suspension was adjusted using a photometer to an optical density of 10 at a wavelength of $600 \mathrm{~nm}$ and then used for inoculation. Only one eye from each animal was infected, with certain non-infected eyes analyzed to serve as controls. Eyes were either not infected or infected, and the infected eyes were either treated or not treated (uninfected/untreated, $\mathrm{n}=6$ eyes; infected/treated, $\mathrm{n}=11$ eyes; infected/untreated, $\mathrm{n}=8$ eyes).

Twenty-four hours post infection the animals were treated under deep anesthesia as described above. For NO treatment, the infected eyes were exposed to gas (Praxair Deutschland $\mathrm{GmbH}$, Düsseldorf, Deutschland) consisting of nitrogen and NO (200 ppm) for $30 \mathrm{~min}$ under anesthesia. A gNO stream with a velocity of $5 \mathrm{~m} / \mathrm{sec}$ was directed to each eye using a nozzle. This nozzle was placed at a distance of $1 \mathrm{~cm}$ from the eye (Fig. 1). Three days after the infection (in two cases, 7 days after the infection to assess whether treatment required $>3$ days to develop an effect) the animals were sacrificed by cervical dislocation.

Histology and evaluation. Their eyes were enucleated, histologically processed, and stained with hematoxylin and eosin. From each eye, five subsequent sections (thickness, $5 \mu \mathrm{m}$, with a distance of $50 \mu \mathrm{m}$ from each other) from the center of the cornea (middle of the pupil) were obtained for evaluation (Fig. 2). The maximum corneal thickness was measured (CellSens Standard 1.8.1; Olympus Deutschland GmbH, Hamburg, Germany) and the mean value was taken. The severity of hypopyon was evaluated with a self-defined score, from 0 to 3 ( 0 , no hypopyon; 1, mild hypopyon; 2, moderate hypopyon; 3, severe/massive hypopyon; Fig. 3).

Statistical analysis. Statistical analysis was performed using GraphPad Prism 5 (GraphPad Software, Inc., La Jolla, CA, USA). The Mann-Whitney U test or the $\chi^{2}$ test were used 
A

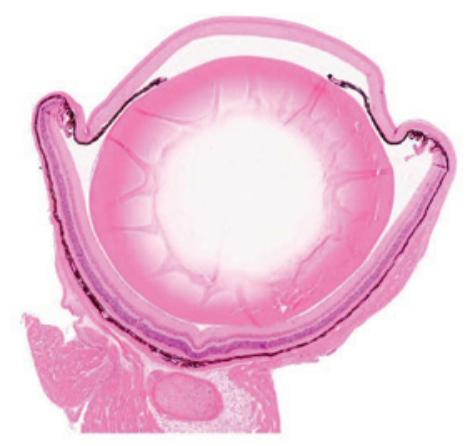

B

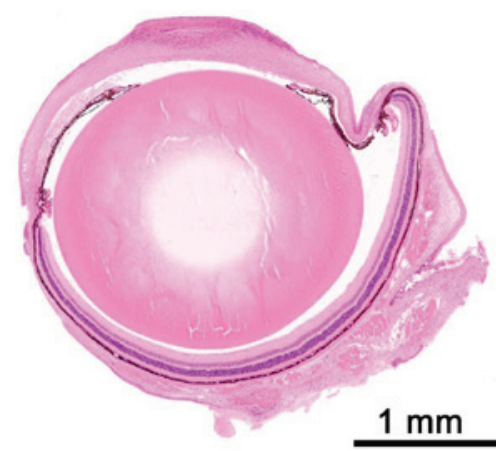

Figure 3. Examples for the severity of hypopyon, stained with hematoxylin and eosin. Score (A) 0 and (B) 1.

A
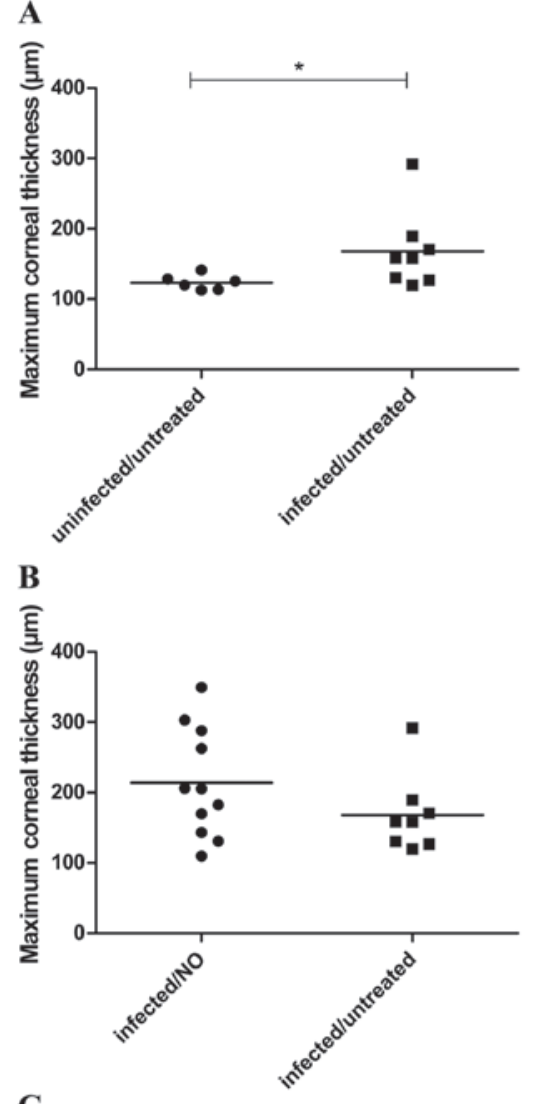

C

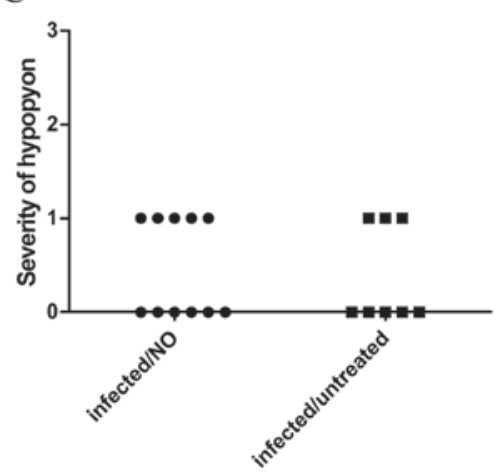

Figure 4. (A and B) Comparison between maximum corneal thickness in the different groups (infected/treated eyes, $\mathrm{n}=11$; infected/untreated eyes, $\mathrm{n}=8$; uninfected/untreated eyes, $\mathrm{n}=6$ ). The bars represent mean values. ${ }^{*} \mathrm{P}<0.05$. (C) Comparison of the severity of hypopyon in NO-treated and untreated infected mice. Each point or square presents the mean value of one mouse. $\mathrm{NO}$, nitric oxide. for inferential statistics. Mean values and standard errors of the mean (SEM) were calculated and two-sided $\mathrm{P}<0.05$ was considered to indicate a statistically significant difference.

\section{Results}

Maximum corneal thickness as an indication of corneal inflammation. Concerning the maximum corneal thickness, the experiments resulted in mean values of $124 \pm 4 \mu \mathrm{m}$ $($ mean \pm SEM) for uninfected/untreated, $168 \pm 20 \mu \mathrm{m}$ (mean \pm SEM) for infected/untreated and $214 \pm 23 \mu \mathrm{m}$ (mean \pm SEM) for infected/treated animals (Fig. 4A and B). The infection led to a significant increase in the maximum corneal thickness. A significant influence of the gNO treatment was not identified for maximum corneal thickness.

Occurrence and severity of hypopyon. Similarly, a significant influence of the gNO treatment was not identified with regard to the severity of hypopyon (Fig. 4C).

\section{Discussion}

Treatment with gNO is a novel and innovative approach as no resistance has yet been identified for this bactericidal mechanism. This initial in vivo study did not indicate an anti-inflammatory effect using gNO. In a previous study regarding bacterial pneumonia that was induced by $P$. aeruginosa, no influence of NO treatment was observed on the inflammation parameters, which was consistent with the present results (9). By contrast, the authors identified a marked reduction in the bacterial load of pseudomonades. The present study hypothesized that i) bacteria were killed and ii) corneal inflammation was reduced following gNO treatment. It might be a similar situation in our keratitis model as in the pneumonia model described in the study by Miller et al (9) where inflammation was not reduced in contrast to the bacterial load. The bacterial load was not determined in the present study, as bacterial load can only be determined in the whole globes, but not separately within the cornea alone, due to the small structure of the murine cornea. However, the hypothesis remains that gNO application kills bacteria within the cornea, but alternative experimental approaches are required. Another aspect of the study was the technical administration of gNO treatment. This treatment strategy was established specifically 
for the current study. The gas application could be improved in future to ensure that a constant gNO concentration is applied to the surface of the cornea, for example, using an eye chamber, although this may be difficult due to the size of the mouse eye.

In conclusion, the aim of the current study was to evaluate gNO application as a treatment for experimental keratitis; this is the first study, to the best of our knowledge, to attempt to treat keratitis using this method. No anti-inflammatory effects were observed; however, this method should be investigated further to evaluate the bactericidal effects in vivo.

\section{Acknowledgements}

The authors would like to thank Ms. Ann Soether for language editing and Ms. Tina Wiesen-Philipps for help with the manuscript.

\section{References}

1. Ong HS and Corbett MC: Corneal infections in the 21st century. Postgrad Med J 91: 565-571, 2015.

2. Lorenzo-Morales J, Khan NA and Walochnik J: An update on Acanthamoeba keratitis: Diagnosis, pathogenesis and treatment. Parasite 22: 10, 2015.
3. Young G, Young AG and Lakkis C: Review of complications associated with contact lenses from unregulated sources of supply. Eye Contact Lens 40: 58-64, 2014.

4. Girgis DO, Sloop GD, Reed JM and O'Callaghan RJ: Susceptibility of aged mice to Staphylococcus aureus keratitis. Curr Eye Res 29: 269-275, 2004.

5. Marquart ME: Animal models of bacterial keratitis. J Biomed Biotechnol 2011: 680642, 2011.

6. Makdoumi K, Mortensen J, Sorkhabi O, Malmvall BE and Crafoord S: UVA-riboflavin photochemical therapy of bacterial keratitis: A pilot study. Graefes Arch Clin Exp Ophthalmol 250: 95-102, 2012

7. Stachon T, Wang J, Song X, Langenbucher A, Seitz B and Szentmáry N: Impact of crosslinking/riboflavin-UVA-photodynamic inactivation on viability, apoptosis and activation of human keratocytes in vitro. J Biomed Res 29: 321-325, 2015.

8. Wu MF, Stachon T, Wang J, Song X, Colanesi S, Seitz B, Wagenpfeil S, Langenbucher A and Szentmáry N: Effect of keratocyte supernatant on epithelial cell migration and proliferation after corneal crosslinking (CXL). Curr Eye Res 41: 466-473, 2016.

9. Miller CC, Hergott CA, Rohan M, Arsenault-Mehta K, Döring G and Mehta S: Inhaled nitric oxide decreases the bacterial load in a rat model of Pseudomonas aeruginosa pneumonia. J Cyst Fibros 12: 817-820, 2013.

10. Ghaffari A, Jalili R, Ghaffari M, Miller C and Ghahary A: Efficacy of gaseous nitric oxide in the treatment of skin and soft tissue infections. Wound Repair Regen 15: 368-377, 2007. 